Except for the above investigations on liver mitochondria, purified xanthine oxidase and yeast lactic dehydrogenase were studied as to their reducibility of TTC and INT using direct spectrophotometric assay method. It was revealed that both of the enzymes began to reduce TTC when oxygen concentration of the solution was decreased to $0 \%$, but that they started reducing INT with no relation to oxygen concentration of the solution.

It was thus concluded that TTC was reduced by mitochondria at the flavoprotein level after the oxygen concentration of the solution was decreased, even in the case of open vessel reaction, to $0, \%$ by the oxygen uptake of mitochondria, and that cyanide and Antimycin A inhibition of succinate TTCreductase was a spurious or pretended inhibition at the cytochrome oxidase level.

It was also suggested that TTC and INT were reduced by not only flavoprotein of mitochondria but also xanthine oxidase and yeast lactic dehydrogenase in accordance with the reduction oxidation potential theory.

\title{
Discussion
}

Dr. Ogawa :

1. Did you study with any other tetrazolium salts than TTC ?

2. Of Nachlas's opinion, it is the essentials that different kinds of tetrazolium salts accept electron through different level of electron transfer system. Dr. Otori did not discuss about this problem.

Dr. Otori :

The present study has shown that TTC is not reduced at the cytochrome oxidase level. Other tetrazoles will be examined in future.

Dr. Mizutani :

1) Did you get any findings with the addition of amytal into the reaction mixtures ?

2) About lactic dehydrogenase, we must be cautious to the different biological characters between yeast and animals, i. e. DPN-independent and dependent.

Dr. Otori :

I have not examined the action of amytal.

\section{Studies on the metachromasia with cyanine dyes}

\author{
Akira OKADA \\ The Department of Pathology, Tuberculosis Research \\ Institute, Kyoto University, Kyoto, \\ (chief : Prof. H. Takamatsu)
}

On the study of the so-called metachromaisa most work has been actually done with thiazine dyes especially toluidine blue or azure A. However, it has been known that many other dyes give metachromatic effect on the specialized chromotropes. 
In 1954, Schiebe and Schauer described a metachromatic effect of pseudoisocyanine on histological sections. This report is dealing with study on metachromasia with many new cyanine dyes for their usefulness in histochemistry and investigation for fundamental matters of metachromasia.

\section{Experiment}

Of the cyanine series 112 dyes were tested in this studies. Complete list of the dyes will be mentioned in another paper. These dyes were synthesized and furnished by Japanese Research Institute for Photosensitizing Dyes co., LTD, 2 dyes of monomethine group, 26 of trimethine group, 12 of pentamethine group, 39 of trinucleous group and 33 of styryl group. Formaline fixed paraffin sections of bronchial cartilage, navel string, various mucous tissues were used for the staining test. Moreover, metachromatic phenomena of diluted dye solutions by addtion of chondroitin sulfate were examined by means of Beckmann DU photoelectric spectrophotometer.

\section{Results and Discussion}

1) Monomethine group : 2 dyes of the group are negative in metachromatic staining.

2) Trimethine group

(a) thiazole: three dyes ( $\mathrm{NK} 535, \mathrm{NK} 77, \mathrm{NK} 1071$,) were tested and all three dyes reacted as metachromatically. NK 77 gave the most effective color change from red to orange, and NK 1071 has a similar molecular structure to NK 77 but does not show any distinct metachromatic reaction on tissue sections, although its metachromatic shift revealed almost the same figure as that of NK 77 (Fig 1,2,). Probably, there are two reasons in this case. The minimum difference of the wave lengths which leads to color change varies

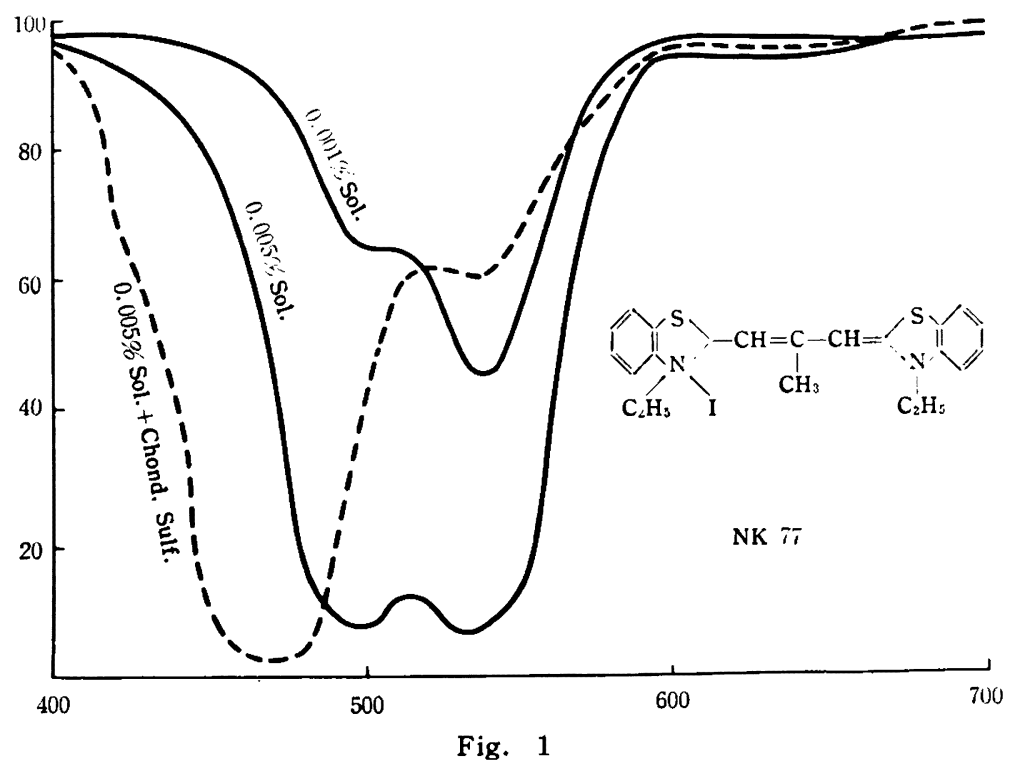




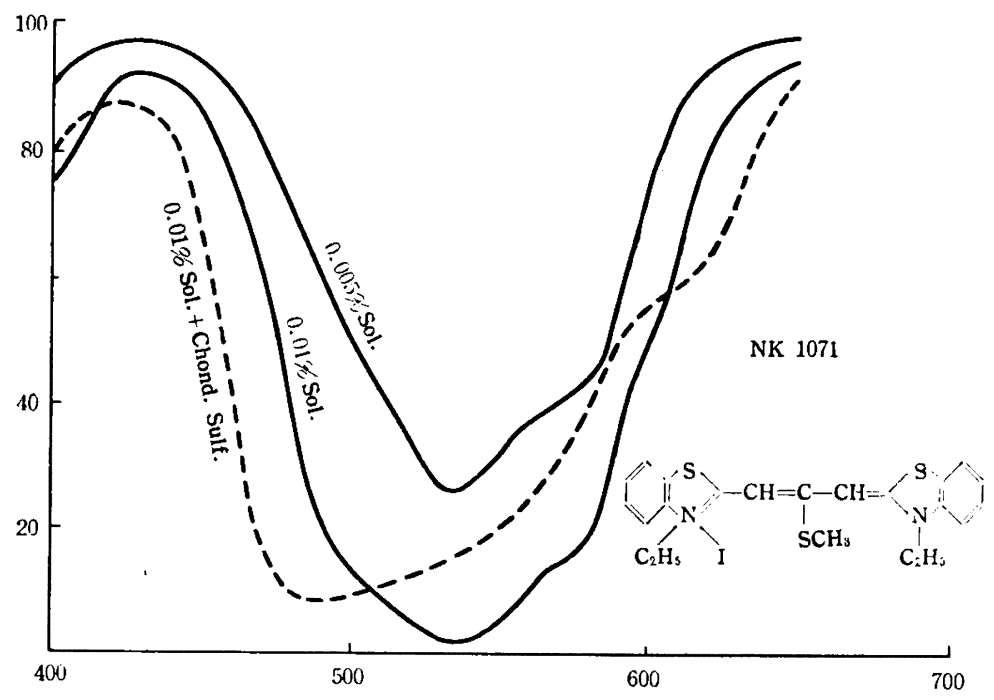

Fig. 2

in individual wave length (This is called difference limen). W. Kelly denoted maximal photopic sensitivity of the eyes as the visual factor for metachromatic phenomenon in study of toluidine blue and methylene blue, probably, the difference limen plays more important role for the case. The metachromatic shift of NK 77 transmitted to the most sensitive region of the difference limen, against this it did not occur in case of NK 1071. Another reason may be the irregularity of the metachromatic curve of NK 1071 . NK 535 is not useful in metachromatic experiment.

(b) chinoline group : NK 3, NK 60 and NK 537 showed characteristic me-

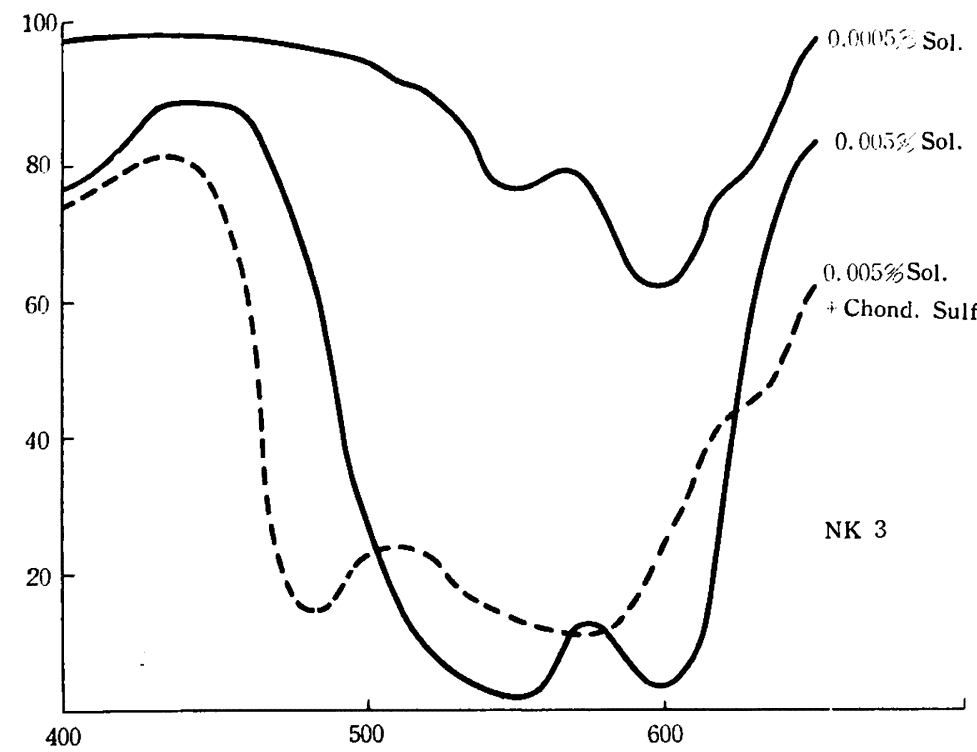

Fig. 3 
tachromatic staining respectively. NK 3 is the dye which has been used for histological or hematological staining since reported as the name of "Illuminol R" staining by S. Hatano and H. Takamatsu'). By addition of chondroitin sulfate in the dye-solution, the purple color will change to blue (Fig 3). If the solution is buffered in range at $\mathrm{pH} 3$ to 4 , addtion of chondroitin sulfate caused color change from blue to purple. In generall, the dyes of the chinoline group are inferior to the thiazole dyes in metachromatic ability.

3) Pentamethine group : NK 193 and NK 210 showed metachromatic staining. NK 210 solution is most sensitive to light, and the color fade out in about 10 minutes.

4) Trinucleous group; Koha 12, 48, 94 and 60 showed metachromatic reaction. Koha 12 and 48 are the dye of thiazole group but Koha 94 and 60 are chinoline containing dyes. NK 79 is non-metachromatic dye and the aqueons solution revealed green color. (fig 4 ).

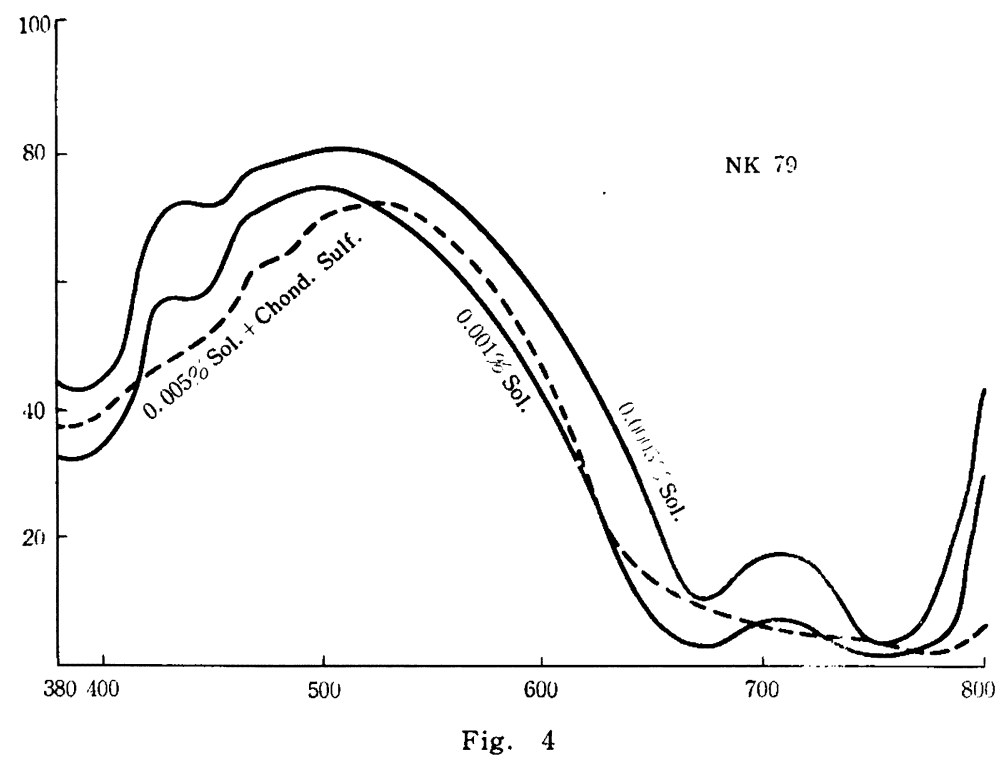

5) Styryl group : Koha 115 and 167 give metachromatic reaction and showed rather different staining from other dyes.

\section{Summary}

1) Of new cyanine dyes 112 kinds were tested for metachromatic staining and the 16 dyes showed metachromatic reaction on histological sections and also proved so in itro.

2) NK 77 and NK 193 are useful dyes for metachromatic staining on tissue sections.

3) The metachromatic phenomenon of the dye solution and metachromatic staining on the tissue sections are compared and the differences between them are discussed. 


\title{
References
}

1. John W, Kelly., The use of metachromasy in Histology, cytology and Histochemistry., Acta. Histochem. 5 (Supple 1) 1958. 2. T.H. Schiebler, S. Schiessler., Uber den Nachweiss von Insulin mit den metachromatisch reagierenden Pseudoisocyanine., Histochem. 1,6, (Schluss) Heff 1959. (Cheibe G., U A, Schauer, Uber den metachromatischen Geweben. S-B. Bayer Akad. wiss. 1954. 14) 3. K. Banno et al. Lists of Photosensitizing dyes (1959) Bulletin of Nippon Kankosikiso Kenkyushio Vol 15. 4. S. Hatano and H. Takamatsu ; Tokyoiji Shinshi, No. 2934. 1935.

\section{Discussion}

Dr. Nagata : It is desirable to apply micro-spectrophotometry of stained tissue sections for the study of metachromasia.

\section{On the enzyme cytochemistry and electron microscopy}

\author{
Tadao Mrrsur \\ Department of Anatomy, School of Medicine Keio University, Tokyo
}

There have been some attempts to apply electron microscope to recognize the products of certain enzymatic cytochemical reaction in order to get better resolutions than those possible with the light microscope. For these studies several methods can be pointed out as follows :

1) Replica. In blood smear technique the replica method can be used to see the reaction end product bound to the cell surface.

2) New deposit. In thin sectioning techniques, enzyme reaction product can be recognized as a new deposit in cytoplasm. This method has been most widely ued in enzyme cytochemistry.

3) Increase of density. Some granules may be relatively electron opaque in normal condition. When a certain enzyme reaction occurs in these granules and the end product is bound to them, the localization of enzyme can be recognized as materials with increased density in the granules under the electron microscope.

4) Comparison between results by light microscope and those by electron microscope. One can recognize by light microscope whether or not a certain enzyme reaction occurs in cells. Then, it is easy to find out what cellular structure changes in positive reaction at the level of electron microscopy.

5) Cell fractionation technique. A definite fraction of cell homogenate is examined by both biochemical and electron microscopic methods. Thus, it can be concluded that a fraction containing high activity of a certain enzyme corresponds to a certain cellular component recognizable under the electron microscope. 